\title{
Evaluation of the Capacitance and Charge Distribution for Conducting Bodies by Circuit Modelling
}

\author{
Dhamodaran Muneeswaran1, Dhanasekaran Raghavan' \\ ${ }^{1}$ Department of Electronics and Communication Engineering, Syed Ammal Engineering College, \\ Ramanathapuram, India \\ ${ }^{2}$ Department of Electrical and Electronics Engineering, Syed Ammal Engineering College, Ramanathapuram, \\ India \\ Email:dhamodaran@syedengg.ac.in,rdhanashekar@syedengg.ac.in
}

Received 6 March 2016; accepted 22 April 2016; published 26 April 2016

Copyright (C) 2016 by authors and Scientific Research Publishing Inc. This work is licensed under the Creative Commons Attribution International License (CC BY). http://creativecommons.org/licenses/by/4.0/ (c) (i) Open Access

\section{Abstract}

This paper presents a numerical analysis for computation of free space capacitance of different arbitrarily shaped conducting bodies based on the finite element method with triangular subsection modeling. Evaluation of capacitance of different arbitrary shapes is important for the electrostatic analysis. Capacitance computation is an important step in the prediction of electrostatic discharge which causes electromagnetic interference. We specifically illustrated capacitance computation of electrostatic models like unit cube, rectangular plate, triangular plate, T-shaped plate, sphere and two touching spheres. Numerical data on the capacitance of conducting objects are presented. The results are compared with other available results in the literature. We used the COMSOL Multiphysics software for the simulation. The models are designed in three-dimensional form using electrostatic environment and can be applied to any spacecraft circuit modeling design. The findings of this study show that the finite element method is a more accurate method and can be applied to any circuit modeling design.

\section{Keywords}

Capacitance, Spacecraft Circuit Modeling, Electrostatic Analysis, Electrostatic Discharge, Finite Element Method

\section{Introduction}

The calculation of electrical capacitance of different arbitrary shapes, like unit cube, rectangular plate, triangular 
plate, T-shaped plate, sphere and two touching spheres, can be considered as significant objects for spacecraft surface charging design. The capacitance is a very important circuit parameter needed for the analysis of electrostatic discharge (ESD). Generally, the spacecraft geometry is considered as a square and the capacitance is being computed approximately. The external surface of the spacecraft design depends on how efficiently a physical structure has been modeled. A well-designed model not only enables conducting a potential study, but also reduces the number of iterations associated with the model. This study gives a complete insight into the properties of devices and circuits, including transmission, emission, electrostatic effects, etc. The problems related to electromagnetic field do not have a systematic solution, and a mathematical approach is essential. In addition, the studies involving electromagnetic field are usually complex and require a very good working knowledge.

The evaluation of capacitance of different arbitrary shapes is important in Computational Electromagnetics (CEM). It deals with the modeling of the interaction between the electromagnetic fields and the physical objects [1] [2]. Compared to the Finite Difference Methods (FDM) and Boundary Element Methods (BEM), the finite element methods provide additional elasticity for local mesh refinement, additional rigorous convergence analysis, additional selections of effective iterative solvers for the secondary linear systems and more elasticity for handling the nonlinear equations. The FEM [1]-[3] is a standard tool for solving the differential equations in electromagnetics. It is also one of the most preferred methods in engineering owing to its significant ability to deal with complex geometries. This paper presents the FEM based analysis for the computation of capacitance and charge distribution of a three dimensional spacecraft circuit modeling. The charge density of the satellite body represented by metallic cube and solar panel consisting of two rectangular plates to the potential on the surface of these bodies is solved. In order to apply the FEM, the surfaces of the metallic bodies are discretized using uniform triangular subsections. The validity of this analysis has been established by comparing the data on capacitance available in the literature for a metallic cube with metallic plates with the data on capacitance computed by the present method for similar structures.

In this paper, the capacitance of the different geometrical assemblies was achieved by subdividing the structure into triangular subsections. The disadvantage of rectangular subsections is that it will not exactly fit into the any arbitrarily shaped geometry. In order to avoid the disadvantage, triangular patch modeling had been in use to perfectly model the arbitrarily shaped surfaces encountered in practical situations [4]-[6]. The Finite Element Method (FEM) is a simpler and easier method compared to other techniques. This method is suitable for solving differential equations and utilizes a more powerful and useful numerical technique for handling the electromagnetic analysis, including difficult geometries [7]-[9] and inhomogeneous media. However, an accurate understanding of the computed results [10]-[12] is essential. It is more important to ensure that the implemented models can be applied to the actual problem to be solved, and the results can be obtained with sufficient speed and accuracy.

\section{FEM Modeling}

\subsection{Expression of Capacitance}

The FEM is a simpler and easier method compared to other techniques. This method is suitable for solving differential equations and utilizes a more powerful and useful numerical technique for handling the electromagnetic analysis including difficult geometries and inhomogeneous media. An efficient and exact computer model of various electromagnetic field problems, including spacecrafts, is made possible using modern high-speed computers and well-developed mathematical techniques. This model enables a spacecraft designer to visualize the targeted spacecraft on the desktop, thereby providing more information in many cases than can ever be measured in the laboratory. The turnaround time required to obtain the spacecraft properties after varying the spacecraft shape is usually calculated in minutes or hours by computer model. The designer can adjust the spacecraft by modifying certain specific parameters of the simulation model. The precision of the existing mathematical model is often such that only a small degree of adjustment is required. However, an accurate understanding of the computed results is essential. It is more important to ensure that the implemented models can be applied to the actual problem to be solved, and the results can be obtained with sufficient speed and accuracy [7].

FEM is well suitable for arbitrary shapes. The simple model of the FEM is based on the behavior of a function, which may be complex when viewed from an enormous region while a simple evaluation may be appropriate for a minor subregion. The entire region is separated into non overlapping subregions called as finite element, and 
the function of each element is approximated by the algebraic expression [8]. In addition, the algebraic representations provide continuity of the function. The efficient generalization of the method makes it possible to build general-purpose computer programs for solving a wide range of difficulties.

The expression of capacitance can be introduced by applying the FEM, i.e., charges and potentials in any system of conductors that create an electric field. Depending on the nature of the system of conductors measured, the capacitance of a solitary conductor, the capacitance between two conductors, and the capacitance in a system of many conductors can be distinguished. The capacitance of the surface can be computed from

$$
C=Q / V \text {. }
$$

where $Q$ is the charge of the conductor (Coulomb),

$V$ is the potential of the conductor (Voltage).

Calculating the capacitance of a simple system like a sphere is important in spacecraft design. Analytical expression for the sphere is denoted by the following equation [9].

The capacitance of the sphere can be computed from

$$
C=4 \pi \varepsilon_{0} a
$$

where $\varepsilon_{0}$ is the permittivity of free space $\left(=8.854 \times 10^{-12} \mathrm{~F} / \mathrm{m}\right)$,

$a$ is the radius of the sphere (meter)

The potential $V_{e}$ is calculated by

$$
V_{e}(x, y)=a+b x+c y
$$

For a triangular element, $a, b$, and $c$ are constants.

A typical triangular element is putting in place of the element governing equations.

The potential $V_{e 1}, V_{e 2}$ and $V_{e 3}$ are calculated by

$$
\begin{aligned}
V_{e 1} & =a+b x_{1}+c y_{1} \\
V_{e 2} & =a+b x_{2}+c y_{2} \\
V_{e 3} & =a+b x_{3}+c y_{3}
\end{aligned}
$$

Similar transformation matrices can be achieved when higher order plane elements are used. The shape functions are calculated in truss element nodes with known coordinates. Typically, these coordinates are agreed in the global coordinates while the shape functions of the plane elements are agreed in the natural coordinate systems. The surface to be analyzed is divided into $N$ number of triangular subsections. The geometry of the reference element is mapped into the geometry of the source triangle using geometrical transformation functions. The parametric coordinates of $\xi$ and $\eta$ in the reference triangle can be mapped into a global coordinates of $x$ and $y$. For triangular elements, the global coordinates $(x, y)$ and the natural coordinates $(\xi, \eta)$ are given by

$$
\begin{aligned}
& x=N_{1} x_{1}+N_{2} x_{2}+N_{3} x_{3} \\
& y=N_{1} y_{1}+N_{2} y_{2}+N_{3} y_{3}
\end{aligned}
$$

where $N_{1}, N_{2}$ and $N_{3}$ are the geometrical transformation function, in which $x_{i}, y_{i}(i=1,2,3)$ represents the coordinates of the triangular element nodes and the shape functions have the following expressions represented in the natural coordinate system as:

$$
\left\{N_{1}=\xi, N_{2}=\eta, N_{3}=1-\xi-\eta\right\}
$$

For the known $x, y$ coordinates, the corresponding natural coordinates can be obtained by solving the following system of equations. Using the above expressions, free space capacitance can be calculated [3] [4] and spacecraft potential can be predicted accurately. The expressions are compact. It is easily fit for the simulation software. However, the algorithm takes more time since the model is more complex.

\subsection{Simulation of Unit Cube}

There is no analytical expression for calculating the electrical capacitance of a unit cube. In this section, the finite element method is used for calculating unit cube [12] [13]. Figure 1 shows a unit cube with each side 


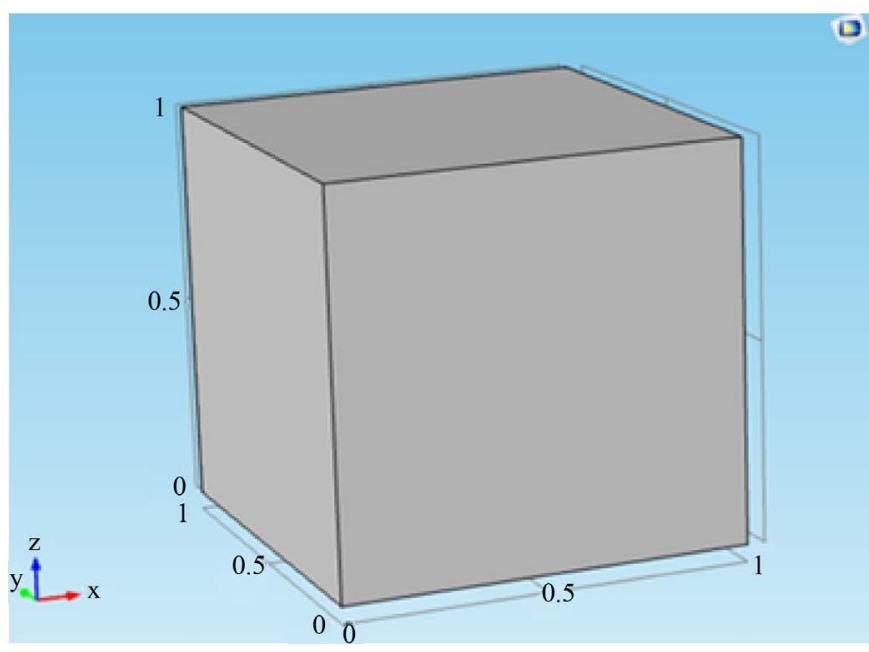

Figure 1. Unit cube with sides of $1.0 \mathrm{~m}(C=66.05 \mathrm{pF})$.

measuring $1.0 \mathrm{~m}$. The model is designed in three-dimensional modeling using electrostatic environment. In the boundary condition of model design, we used ground boundary which is zero potential. For the unit cube, the bottom of the cube is specified as ground with a voltage of $0 \mathrm{~V}$. The top of the cube has a specified voltage of 1 $\mathrm{V}$. It is assumed that the unit cube is made of highly conductive material in which the total resistive value is much lower. When the unit voltage is applied to the object the charge densities near the edge of the bodies [1] [3] are much higher than those far away from the edges.

From the unit cube model, we produced more number of subsections and 2300 domain element in the finite element mesh shown in Figure 2. The potential distribution simulations help to better understand the potential distribution of the metallic object. The modeling produces the finite element mesh with triangular subsections and 1536 boundary elements, which shows that the three-dimensional view of the unit cube with triangular subsections. Figure 3 show that the three-dimensional surface potential distribution of the metallic cube with triangular subsections. The potential distributions of inhomogeneous media of metallic surface are simulated. There is electric potential are uniform between the cube surfaces, but the variances can be seen at the top and bottom of the cube. The potential distribution demonstrates that the topmost of the cube having more current flow compared to bottommost of the cube.

In some cases, the capacitance values are calculated with respect to variation in the number of domain elements and boundary elements. The results are tabulated in Table 1 . The capacitance value $66.05 \mathrm{pF}$ obtained is equated with the value obtained from the earlier result. The results tend to converge, and the deviation in analytical and numerical results decreases. It is clear from the table that the present solver leads to very accurate results. Present method takes less time for the simulation.

In this section, the simulation of the unit cube is analyzed. The findings of this study are in accordance with the available results [6]. According to Wintle [19], a random walk method was used for the computation of unit cube; in which the authors found an error in the method of parallel curves. Since a random walk method uses smaller steps, they have been distributed randomly as well as these methods avoid hidden systematic errors. Similarly, Mascagni and Simonov [18] used Monte Carlo technique for the computation of the capacitance of the unit cube. In order to estimate the computational error; Markov chain version of the central limit theorem was used. Hwang and Mascagni [6] used walk on planes-Monte Carlo Algorithm and the results are published. Compare to other methods, FEM take less time for simulation and more accuracy.

\subsection{Simulation of Rectangular Plate}

In this section, the modeling of rectangular plate by determining capacitance using finite element method is discussed. The capacitance values are calculated for certain cases with respect to the difference in the number of domain elements and boundary elements in rectangular plate. Figure 4 shows rectangularplate with sides of 1.0 $\mathrm{m}$ and $4.0 \mathrm{~m}$. The model is calculated in three- dimensional environment in order to compare the results with some literature available [11]. 


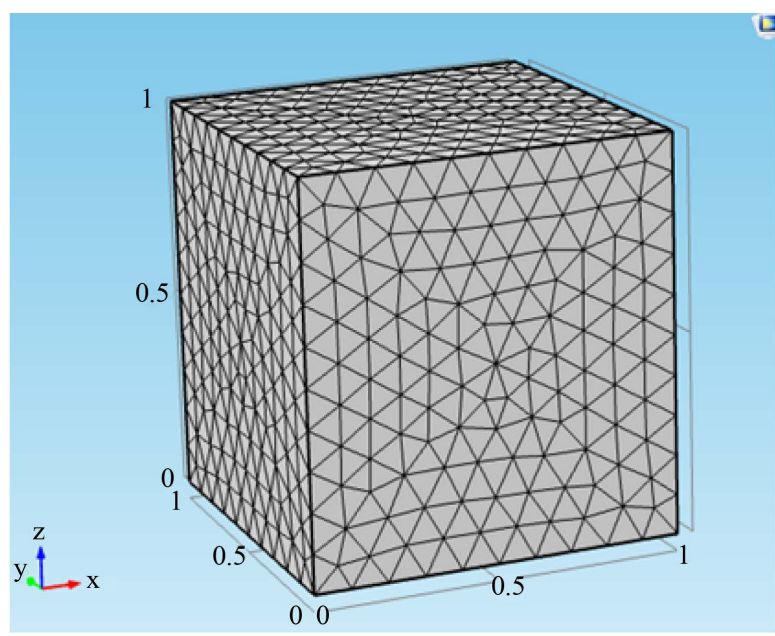

Figure 2. Unit cube with triangular subsection.

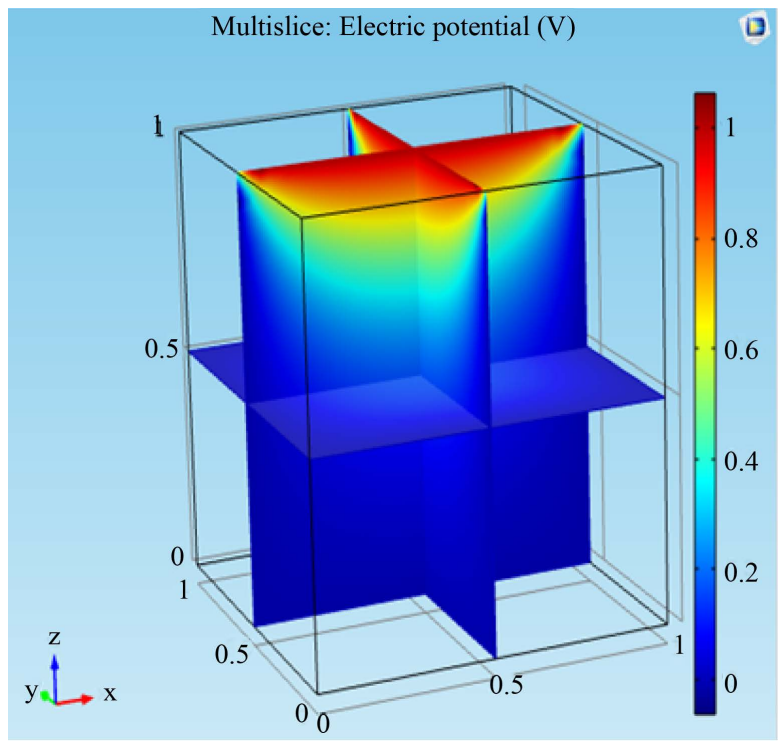

Figure 3. Charge distribution on unit cube.

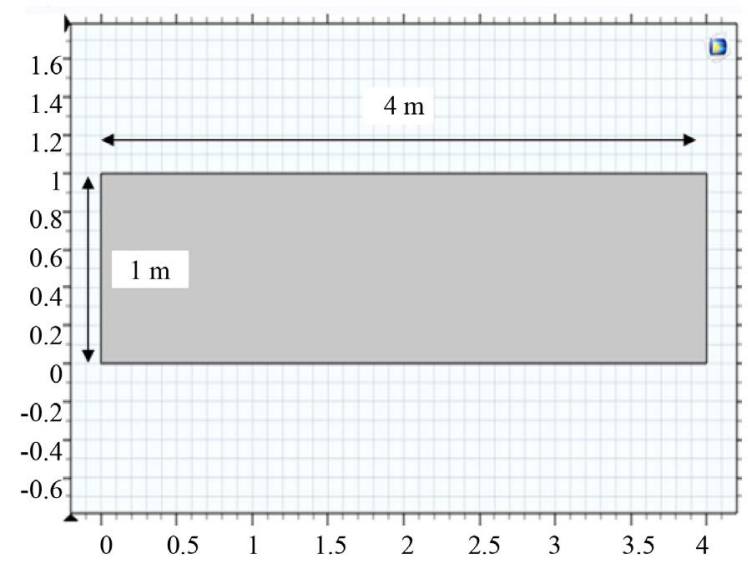

Figure 4. Rectangular plate with sides of $1.0 \mathrm{~m}$ and $4.0 \mathrm{~m}$ $(\mathrm{C}=54.42 \mathrm{pF})$. 
Table 1. Results for the capacitance of the unit cube.

\begin{tabular}{ccc}
\hline Geometry & Proposed Method Capacitance & Method [5] [11] Capacitance \\
\hline Van Bladel [20] & Variation method & $65.56[\mathrm{pF}]$ \\
Read [17] & Refined boundary element method & $66.07[\mathrm{pF}]$ \\
Wintle [19] & Random walk method with variance reduction & $66.06[\mathrm{pF}]$ \\
Mascagni [18] & Random walk on the boundary & $66.07[\mathrm{pF}]$ \\
Hwang [6] & Walk on planes & $66.07[\mathrm{pF}]$ \\
& Proposed method & $66.05[\mathrm{pF}]$ \\
\hline
\end{tabular}

The simulation produces the finite element mesh with triangular subsections and 120 boundary elements as shown in Figure 5(a). It shows the three-dimensional view of the metallic rectangular plates with triangular subsections [12]. Figure 5(b) shows the potential distributions of inhomogeneous media of metallic plates. The potential distribution is constant between the plates, but variances can be seen at the side of the plate [14]. The potential distribution shows that the topmost edge of the plate has more current flow compared to the bottommost of the plate. The results are in good agreement with the earlier results. The capacitance value $54.62 \mathrm{pF}$ is obtained for a total variety of 220 triangular subsections.

\subsection{Simulation of Equilateral Triangle Plate}

The modeling of an equilateral triangle is an important geometrical model for spacecraft circuit modeling design. The capacitance values are calculated with respect to the difference in the number of domain elements and boundary elements in equilateral triangle plate. Figure 6 shows an equilateral triangular plate with each side of $1.0 \mathrm{~m}$. The model is calculated in three-dimensional environments in order to compare the results with some of the other results [15].

The modeling resulted in a finite element mesh with triangular subsections and 80 boundary elements as shown in Figure 7(a). It shows that the three-dimensional view of the metallic plates with triangular subsections [15]. Figure 7(b) shows the potential distributions of inhomogeneous media of metallic plates. The potential distribution is constant between the plates, but variances can be seen at the centre of the plate. The results are in good agreement with earlier results [16] [17]. The capacitance value $23.35 \mathrm{pF}$ is obtained for a total of 173 triangular subdivisions.

Ghosh and Chakrabarty [5] computed capacitance of triangular platebased on method of moment with pulse basic function was used. In this computation, the variation of capacitance with the number of sub-sections of the structure analysed. In that technique, the capacitance was evaluated by dividing the conducting surface into rectangular subsection. However, the resulting equations for the potential coefficients are found to be complicated and these were mainly used for three-dimensional bodies with rectangular surfaces.

\subsection{Simulation of T-Shaped Plate}

In this section, the utility of FEM used to calculate the electrical capacitance of T-shaped plate is discussed. Figure 8 shows a T-shaped platewith a dimension of $3.0 \mathrm{~m}, 1.0 \mathrm{~m}$, and $3.0 \mathrm{~m}$. The model is designed based on three-dimensional modeling using electrostatic environment [18]. T-shaped plate model like 180 domain element of the finite element mesh is shown in Figure 9(a). The charge distribution simulations help to understand the potential distribution of the metallic object [19] [20]. From the model, finite element mesh with triangular subsections and 504 boundary elements were generated. The three-dimensional view of the T-shaped platewith triangular subsections was presented [21].

Figure 9(b) shows the surface potential distribution of the metallic T-shaped plate. The potential distributions of inhomogeneous media of metallic surface are simulated. The free space capacitance values are calculated for some cases with respect to variation in a number of domain elements and boundary elements. The capacitance value obtained is $98.43 \mathrm{pF}$, equated with the value obtained from the earlier results [5]. The results tend to converge and the deviation in analytical and numerical results is reduced [21] [22]. 


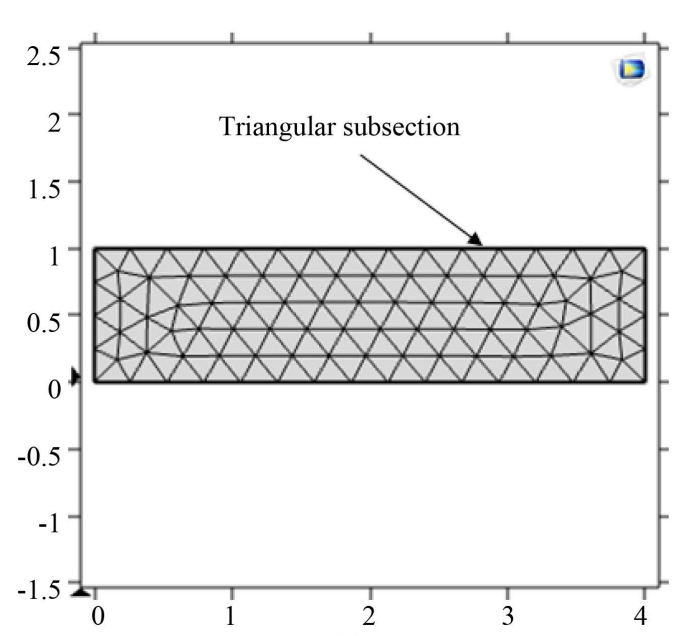

(a)

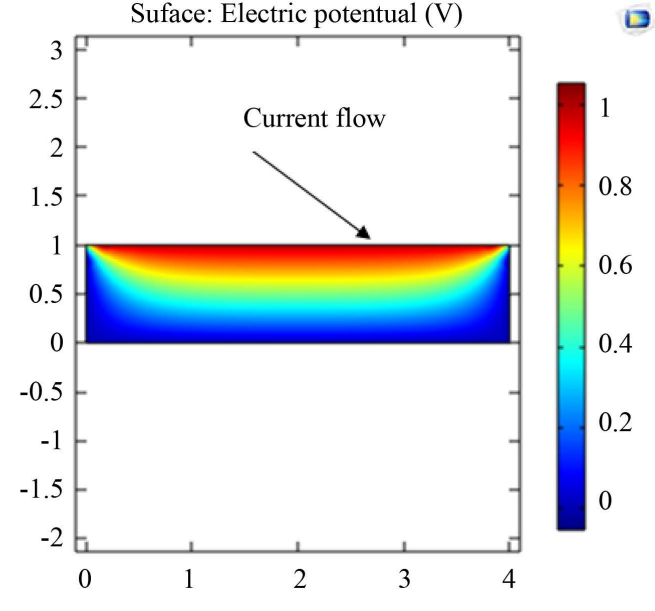

(b)

Figure 5. Rectangular plate model. (a) Rectangular plate with triangular subsection. (b) Charge distribution on a rectangular plate.

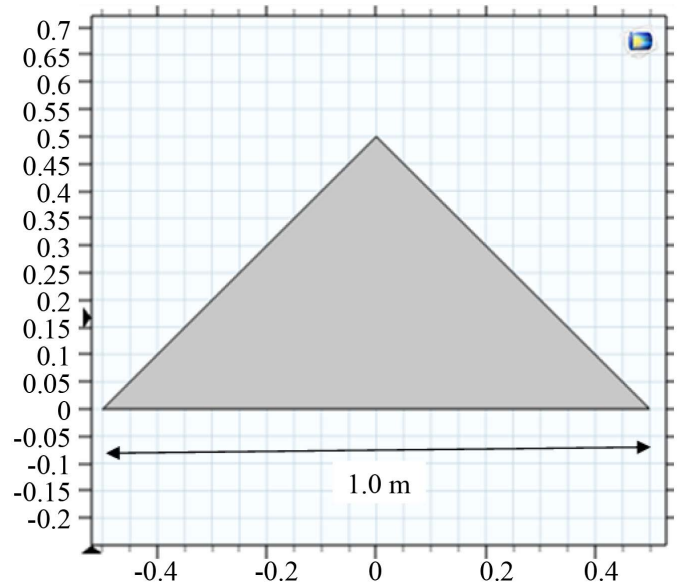

Figure 6. Equilateral triangular plate with each sides of $1.0 \mathrm{~m}(\mathrm{C}=23.35 \mathrm{pF})$.

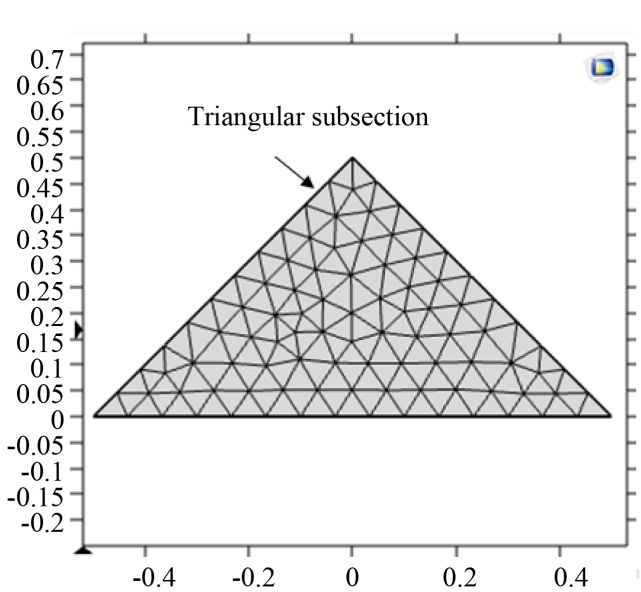

(a)

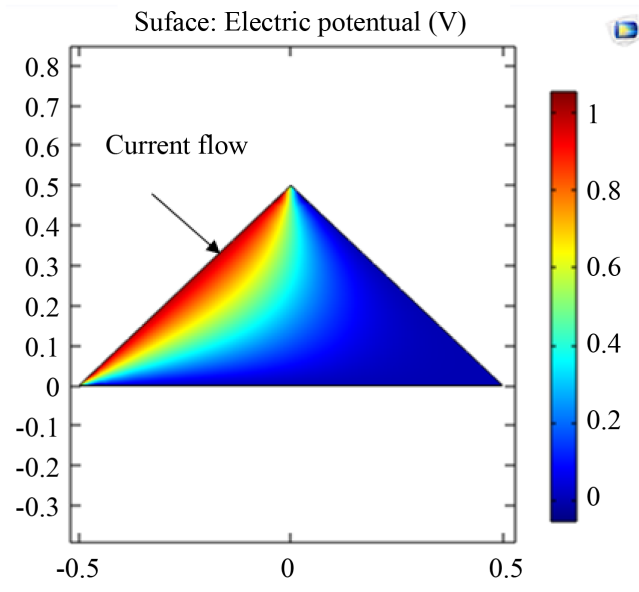

(b)

Figure 7. Triangular plate model. (a) Triangular plate with triangular subsection. (b) Charge distribution on triangular plate. 


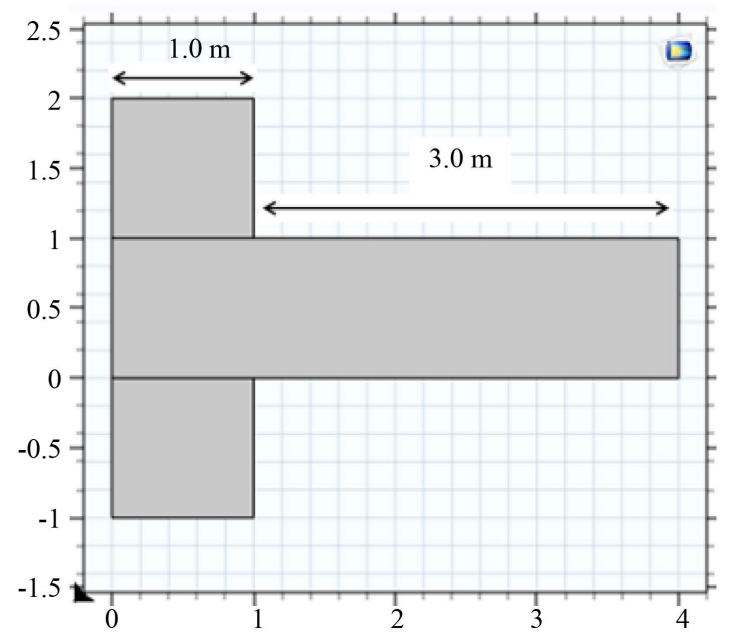

Figure 8. T-shaped plate with sides of $3.0 \mathrm{~m}, 1.0 \mathrm{~m}$, and $3.0 \mathrm{~m} .(\mathrm{C}=98.43 \mathrm{pF})$.

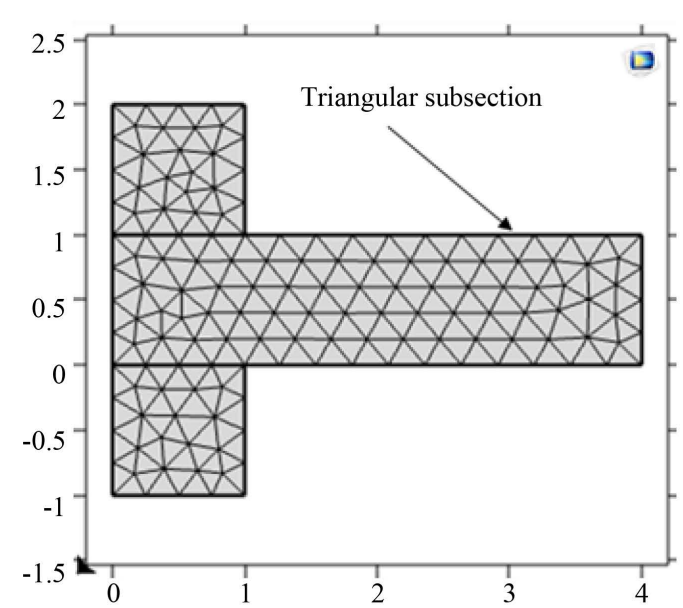

(a)

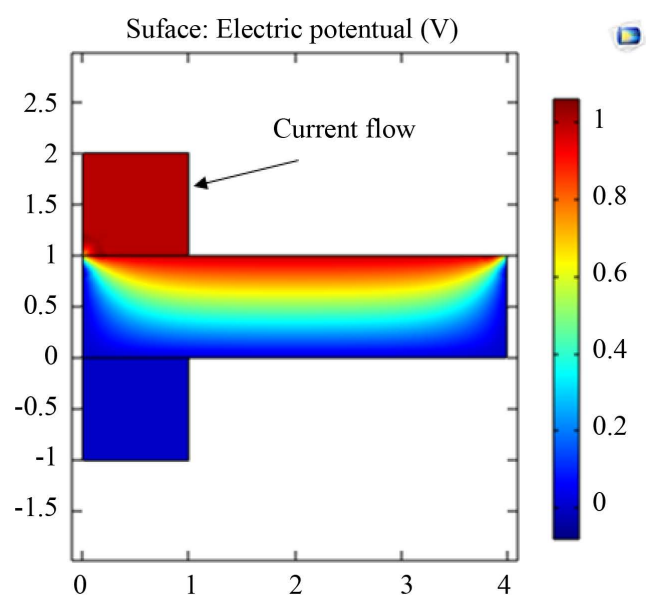

(b)

Figure 9. T-shaped plate model. (a) T-shaped plate with triangular subsection. (b) Charge distribution on Tshaped plate.

\subsection{Simulation of Sphere}

In this section, the FEM is used to calculate the electrical capacitance of sphere. Figure 10 shows a sphere with a radius of $1 \mathrm{~m}$. The model is designed based on three-dimensional modeling using electrostatic environment [23]-[25].

Under the boundary condition of model design, it is possible to produce a number of subsections from the sphere model like 2058 domain element of the finite element mesh and the potential distribution simulations help to better understand the potential distribution of the metallic object. From the model, the finite element mesh with triangular subsections and 504 boundary elements were produced. Figure 11 shows the threedimensional (3D) view of the sphere with triangular subsections.

Figure 12 shows the three-dimensional surface potential distribution of the metallic sphere with triangular subsections.

The capacitance values are calculated for some cases such as with respect to variation in a number of domain elements and boundary elements. The capacitance value obtained, $111.47 \mathrm{pF}$, is equated with the value obtained from the earlier results. The results tend to converge and the deviation in analytical and numerical results is reduced.In this study, the values matched with the available results. In this section, the sphere simulations with different subsections are analyzed. The results obtained in this study correspond with the available results [17]. 


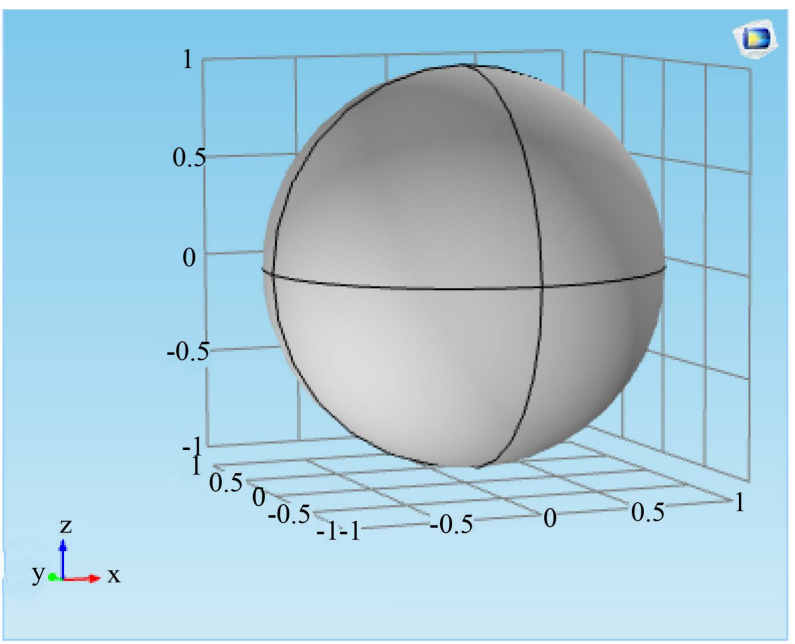

Figure 10. Metallic sphere model.

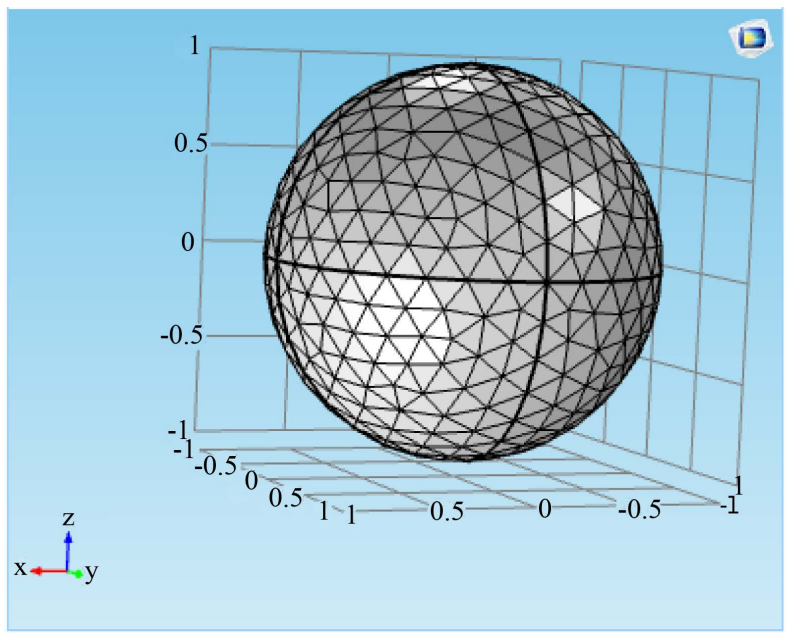

Figure 11. Sphere with triangular subsections.

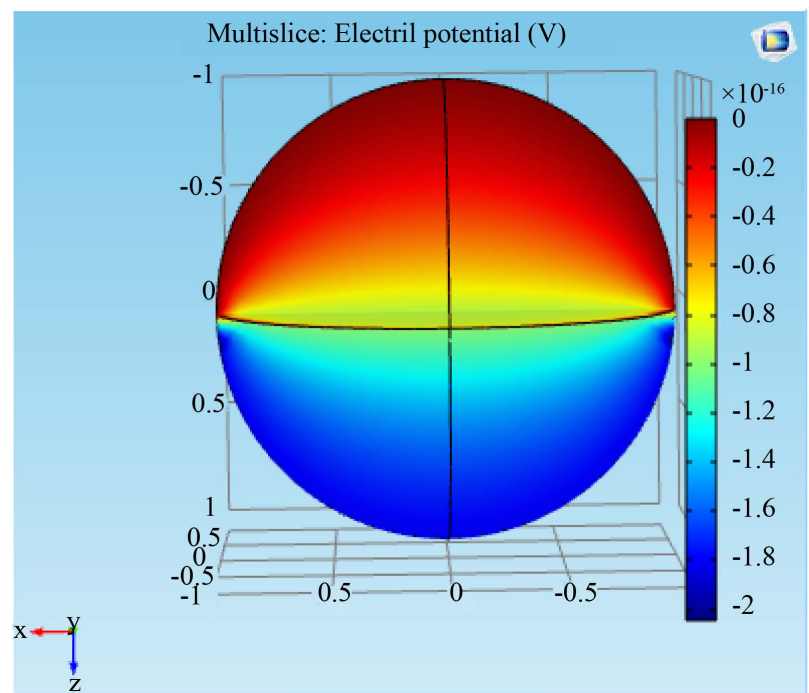

Figure 12. Charge distribution on sphere. 


\subsection{Simulation of Touching Spheres}

In this section, FEM used for calculating the capacitance of two touching spheres is discussed. Touching spheres are important in circuit modeling design. When the two spheres touch, they can be regarded as being fused into one single complex conductor. Figure 13 shows the identical spheres touching together with each having a dimension of $1.0 \mathrm{~m}$ radius. The model is designed based on the three-dimensional modeling using electrostatic environment. It is assumed that the touching spheres are made of a highly conductive material such that their total resistance value is much lower. When the unit voltage is applied to the object, the charge densities of the nearby edge of the bodies are much higher than those that are placed far away from the edges [11].

From the model, the finite element mesh with triangular subsections and 504 boundary elements were produced as shown in Figure 14(a). It shows that the three dimensional view of the two touching spheres with triangular subsections. Figure 14(b) shows the three dimensional surface potential distribution of the metallic spheres with triangular subsections. The potential distributions of inhomogeneous media of metallic surface are simulated [21] [22]. The capacitance value of $154.57 \mathrm{pF}$ is obtained and equated with the value obtained from the earlier results. The results tend to converge and the deviation in analytical and numerical results is reduced.

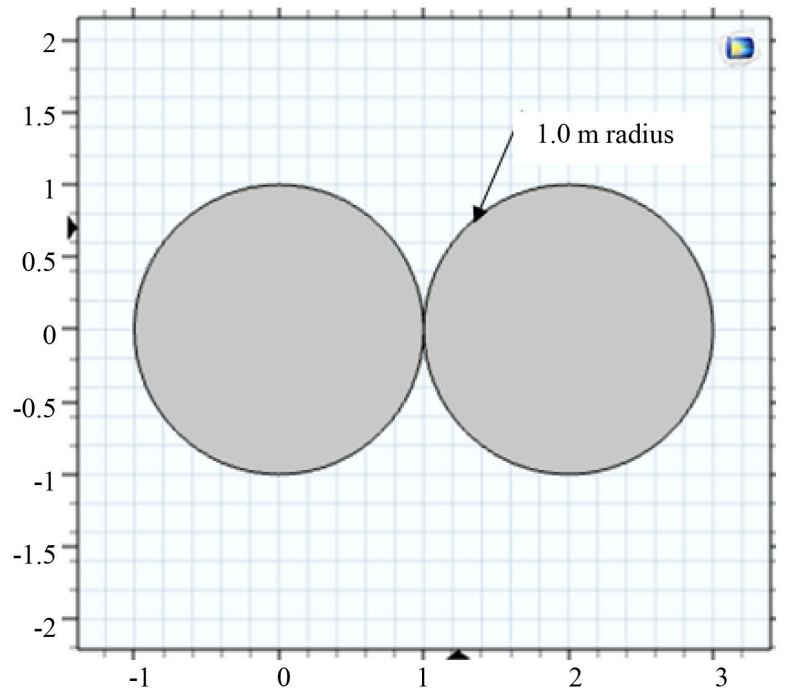

Figure 13. Touching spheres model.

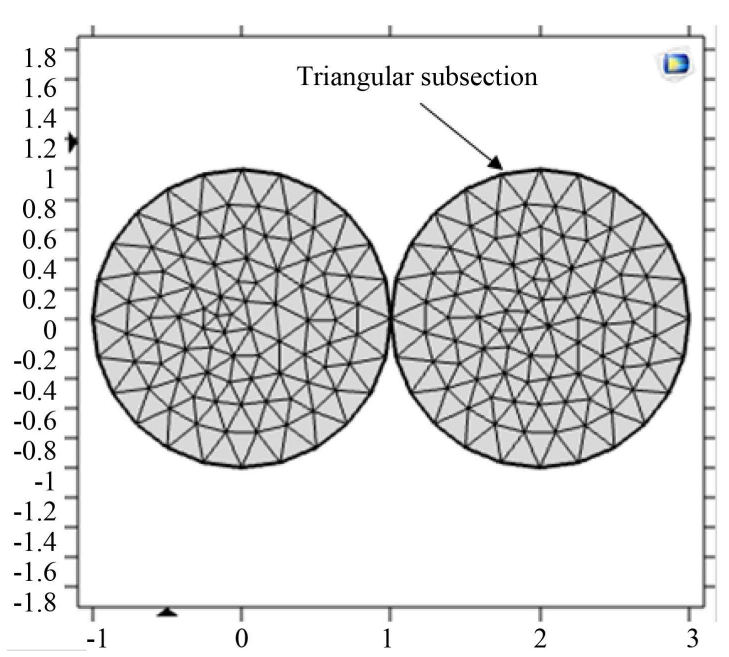

(a)

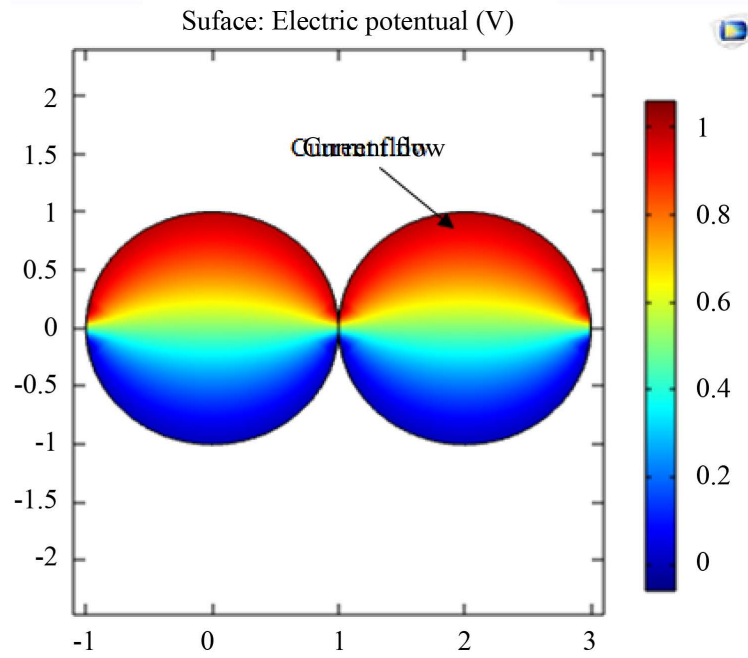

(b)

Figure 14. Touching sphere model. (a) Touching spheres with triangular subsection. (b) Charge distribution. 
Table 2. Comparison of capacitance of different arbitrary shapes.

\begin{tabular}{ccc}
\hline Geometry & Proposed Method Capacitance & Method [5] [11] Capacitance \\
\hline Rectangular plate & $54.62[\mathrm{pF}]$ & $54.73[\mathrm{pF}]$ \\
Triangular plate & $23.35[\mathrm{pF}]$ & $23.13[\mathrm{pF}]$ \\
T-shaped plate & $98.43[\mathrm{pF}]$ & $98.53[\mathrm{pF}]$ \\
Sphere & $111.47[\mathrm{pF}]$ & $111.8[\mathrm{pF}]$ \\
Two touching spheres & $154.57[\mathrm{pF}]$ & $154.8[\mathrm{pF}]$ \\
\hline
\end{tabular}

\section{Results and Discussion}

In the present study, the capacitance of a unit cube was $66.05 \mathrm{pF}$, which is similar to that obtained in other studies. The capacitance of the rectangular plate was found to be $54.62 \mathrm{pF}$, which is similar to capacitance obtained in other studies, i.e., $54.73 \mathrm{pF}$ [5]. Similarly, the capacitance of a triangular plate was computed as $23.35 \mathrm{pF}$, which is similar to that obtained in other studies, i.e., $23.13 \mathrm{pF}$. As well, the capacitance of a T-shaped plate was computed as $98.43 \mathrm{pF}$, which is similar to the one obtained in previous studies, i.e., $98.53 \mathrm{pF}$ [7]. The capacitance of a T-shaped plate was also computed as $98.43 \mathrm{pF}$, which is similar to that obtained in other studies, i.e., $98.53 \mathrm{pF}$. The capacitance in the sphere was computed as $111.47 \mathrm{pF}$, which is similar to that obtained in other studies. i.e. $111.8 \mathrm{pF}$. Moreover, the capacitance of the two touching spheres was found to be $154.57 \mathrm{pF}$, which is similar to the capacitance obtained in other studies, i.e., $154.8 \mathrm{pF}$ [11]. The application of numerical techniques involves the usage of computers [26] and appropriate program packages. The outcome of the present study shows that the FEM is more effective and accurate than the other methods.

The results of five geometries are summarized in Table 2 and compared with the earlier results [5] [11]. Using FEM, more accurate value is achieved. The process outlining the usage of triangular subdivision produces more accurate value. All simulations were performed on a PC with core-i3 processor with $3.1 \mathrm{GHz}$ CPU and 8 GB of RAM. COMSOL results for the capacitance of the model compared with the previous work that investigated numerical method. In this study, the values obtained matched with the available results.

However, this study has certain limitations that have been acknowledged. The simulation was confined to other methods like random walk method and method of moments. Nevertheless, in spite of these limitations, this study provided new insights into the capacitance computation of different conducting bodies.

\section{Conclusion}

FEM has been found to be the most accurate method for evaluating free space capacitances. In the present study, different arbitrary shapes are analysed for electrostatic modelling. The capacitance of different arbitrary shaped conductors like unit cube, rectangular plate, triangular plate, T-shaped plate, sphere and two touching spheres was calculated. Some of the simulations obtained in the study show the usage of FEM with COMSOL Multiphysics software. The results derived from using the software correspond with the results of previous studies. Thus, the method is more suitable for various shapes involved in spacecraft circuit modeling design. This approach is simple and can be applied to any practical shapes of the metallic objects.

\section{References}

[1] Smythe, W.R. (1968) Static and Dynamic Electricity. McGraw-Hill, New York.

[2] Sadiku, M.N.O. (2007) Elements of Electromagnetics. 4th Edition, Oxford University Press, New York.

[3] Dhatt, G. and Touzot, G. (1984) The Finite Element Method Displayed. John Wiley \& Sons, New York.

[4] Jin, J. (2002) Finite Element method in Electromagnetic. Wiley, New York.

[5] Ghosh, S. and Chakrabarty, A. (2008) Estimation of Capacitance of Different Conducting Bodies Using Rectangular Subareas. Journal of Electrostatics, 66, 142-146. http://dx.doi.org/10.1016/j.elstat.2007.11.003

[6] Hwang, C.-O. and Mascagni, M. (2004) Electrical Capacitance of the Unit Cube. Journal of Applied Physics, 95, 3798-3802. http://dx.doi.org/10.1063/1.1664031

[7] Hariharan, V.K. (1991) On the Development of Equivalent Electric Circuit Modeling. PhD Dissertation, IIT, India.

[8] Moghadama, A.A.A., Torabib, K. and Moaveniana, M. (2011) Finite Element Modeling and Robust Control of Fast 
Trilayerpolypyrrole Bending Actuators. International Journal of Applied Electromagnetics and Mechanics, 35, 281-305.

[9] Alad, R.H. and Chakrabarty, S.B. (2013) Capacitance and Surface Charge Distribution Computations for a Satellite Modeled as a Rectangular Cuboid and Two Plates. Journal of Electrostatics, 71, 1005-1010. http://dx.doi.org/10.1016/j.elstat.2013.09.006

[10] Karthikeyan, B., Hariharan, V.K. and Sanyal, S. (2013) Estimation of Free space Capacitance and Body Potential of a Spacecraft for Charging Analysis. IEEE Transactions on Plasma Science, 41, 3487-3491. http://dx.doi.org/10.1109/TPS.2013.2271280

[11] Chow, Y.L. and Yovanovich, M.M. (1983) The Capacitances of Two Arbitrary Conductors. Journal of Electrostatics, 14, 225-234. http://dx.doi.org/10.1016/0304-3886(83)90009-8

[12] Nishiyama, N. and Nakamura, M. (1994) Forms and Capacitance of Parallel Plate Capacitors. IEEE Transactions on Components, Packaging, and Manufacturing Technology: Part A, 17, 477-484. http://dx.doi.org/10.1109/95.311759

[13] Rao, S.M., Glisson, A.W., Wilton, D. and Vidula, B. (1979) A Simple Numerical Solution Procedure for Statics Problems Involving Arbitrary-Shaped Surfaces. IEEE Transactions on Antennas and Propagation, 27, 604-608. http://dx.doi.org/10.1109/TAP.1979.1142171

[14] Rafighi, F., Behrouzinia, S., Khorasani, K., Sabaghi, M. and Marjani, S. (2016) The Electrical Parameters Modeling and Experimentation of Copper Vapor Laser. Circuits and Systems, 7, 23-28. http://dx.doi.org/10.4236/cs.2016.71003

[15] Chakraborty, C., Poddar, D.R., Chakraborty, A. and Das, B.N. (1993) Electrostatic Charge Distribution and Capacitance of Isolated Cylinders and Truncated Cones in Free Space. IEEE Transactions on Electromagnetic Compatibility, 35, 98-102. http://dx.doi.org/10.1109/15.249403

[16] Alad, R.H., Chakrabarty, S.B. and Lonngren, K. (2012) Electromagnetic Modeling of Metallic Elliptical Plates. Journal of Electromagnetic Analysis and Applications, 4, 468-473. http://dx.doi.org/10.4236/jemaa.2012.411065

[17] Read, F.H. (2004) Capacitances and Singularities of the Unit Triangle, Square, Tetrahedron and Cube. COMPEL-The international Journal for Computation and Mathematics in Electrical and Electronic Engineering, 23, 572-578. http://dx.doi.org/10.1108/03321640410510758

[18] Mascagni, M. and Simonov, N. (2004) The Random Walk on the Boundary Method for Calculating Capacitance. Journal of Computational Physics, 195, 465-473. http://dx.doi.org/10.1016/j.jcp.2003.10.005

[19] Wintle, H.J. (2004) The Capacitance of the Cube and Square Plate by Random Walk Methods. Journal of Electrostatics, 62, 51-62. http://dx.doi.org/10.1016/j.elstat.2004.04.005

[20] Van Bladel, J. and Mei, K. (1961) On the Capacitance of a Cube. Applied Scientific Research, Section B, 9, $267-270$. http://dx.doi.org/10.1007/BF02921813

[21] Dhamodaran, M. and Dhanasekaran, R. (2015) Evaluation of Capacitance of Conducting Bodies for Electromagnetic Modeling. Journal of Engineering and Applied Sciences, 10, 2743-2748.

[22] Dhamodaran, M. and Dhanasekaran, R. (2015) Electromagnetic Analysis of Different Metallic Plates. International Journal of Applied Engineering Research, 10, 712-716.

[23] Dhamodaran, M. and Dhanasekaran, R. (2014) Comparison of Computational Electromagnetics for Electrostatic Analysis. International Journal of Energy Optimization and Engineering, 3, 86-100. http://dx.doi.org/10.4018/ijeoe.2014070106

[24] Dhamodaran, M. and Dhanasekaran, R. (2014) Efficient Capacitance Computation for Computational Electromagnetics. IEEE International Conference on Communications and Signal Processing, Melmaruvathur, 3-5 April 2014, 1674-1679. http://dx.doi.org/10.1109/iccsp.2014.6950133

[25] Dhamodaran, M. and Dhanasekaran, R. (2013) Comparison of Capacitance Computation by Different Methods. IEEE International Conference on Communications and Signal Processing, Melmaruvathur, 3-5 April 2013, 73-77. http://dx.doi.org/10.1109/iccsp.2013.6577018

[26] COMSOL Multiphysics, Version 5.0 Release Documentation, 2015. 